\title{
Latent diversity in human concepts
}

\begin{abstract}
Many social and legal conflicts come down to differences in semantics. Yet, semantic variation between individuals and people's awareness of this variation have been relatively neglected by experimental psychology. Here, across two experiments, we quantify the amount of agreement and disagreement between ordinary semantic concepts in the population, as well as people's meta-cognitive awareness of these differences. We collect similarity ratings and feature judgements, and analyze them using a non-parametric clustering scheme with an ecological statistical estimator to infer the number of different meanings for the same word that is present in the population. We find that typically at least ten to twenty variants of meanings exist for even common nouns, but that people are unaware of this variation. Instead, people exhibit a strong bias to erroneously believe that other people share their particular semantics, pointing to one factor that likely interferes with political and social discourse.
\end{abstract}

Key words: Concepts, Metacognition, Individual differences, Ordinary meaning

\section{Statement of Relevance}

Cognitive science has long debated the degree to which common word meanings differ across individuals. Combining empirical data with state of the art modelling techniques, we statistically quantify the number of distinct concepts for 20 words across the population. We find strong evidence that the probability a single concept exists for each word is very small, and the most likely scenario is that roughly ten to twenty concepts exist, even for everyday nouns. These results suggest that fundamental conceptual differences underlie many semantic disagreements in political and social discourse.

\section{Introduction}

Children learn word meanings through experience, and experiences differ between people. This suggests that even when two individuals use the same word, they may not agree precisely on its meaning. Indeed disagreements about meaning can be found in debates about the meaning of terms like "species" (1), "genes" (2), or "life" (3) in biology; "curiosity" (4), "knowledge" (5), or "intelligence" (6) in psychology; and "measurement" in physics (7). Ernest Mach and Albert Einstein even disagreed about what constitutes a 
"fact" (8); in contemporary society, social issues hinge on the precise meaning of terms like "equity" (9), "pornography" (10), "peace" (11), or the "right to bear arms" (12). Such debates often end up in the legal system. For example, in 1893, the U.S. Supreme court decided in Nix v. Hedden that, for tax purposes, a tomato counted as a vegetable, not a fruit, stating that the law followed the "ordinary meaning" (see (13)) of words rather than their botanical meaning.

Despite the frequency with which word meanings are debated, there have been few efforts to understand and quantify such variation in mental representations using tools of cognitive psychology. In the 1970s, Labov (14) examined individual differences in people's classifications in a simple two-dimensional space of stimuli, cups and bowls that varied in height and width. This work found people often disagreed in atypical cases, a finding that holds in other domains (15). Psychometrics developed multidimensional scaling methods (16-18) which account for individual differences, starting in the 1960s (19-24), with recent implementations (25) providing the advantages of generative Bayesian statistical inference $(26,27)$. However, these tools have not been used to study population variation itself. Variation has been documented with amount of training or specialization-for instance, philosophers view "knowledge" differently than other academics and non-academics (28), and specialists often develop a specialized lexicon (29). Relatedly, recent results show that adults (30) and children (31) will sometimes use words without real understanding.

While prior work has modeled individual variation, it has not sought to robustly quantify how many varieties of ordinary concepts exist in the population. A primarily challenge is that there are no complete accounts of human conceptual representation (see, e.g. (32)) and therefore people's representations must be measured indirectly. To accomplish this, we ran two experiments, collecting people's judgements of similarity between concepts and judgements of conceptual features respectively. The similarity experiment asked people to judge whether, for example, a penguin is more similar to a chicken or a whale. Similarities have been seen as foundational to some aspects of meaning, both in classic work $(17,18,33)$ and in more recent semantic representational theories $(34,35)$. The feature experiment first freely elicited features, and then asked a group of participants to rate those on each concept. For example, participants judged whether a penguin was "majestic." We gathered ratings in two domains: common animals and politicians. These domains allows us to characterize diversity for high-frequency nouns, which may be most likely to be shared. We contrast this with politicians, which might vary among individuals with distinct political beliefs, as do concepts and language concerning morality $(36,37)$. We note that similarity judgements and features have well-known limitations, including for example that similarities are sensitive to the respects with which similarity is computed (38-41). However, because we are interested in quantifying diversity, it is not important to the experiment that such features and similarities do not completely characterize people's conceptual knowledge. Differences in features and similarities still indicate that there are some underlying conceptual differences.

Importantly, we asked participants to make the same similarity ratings and feature judgements multiple times, allowing us to determine how reliable and stable the ratings were. This was important for our primary analysis because it allowed us to include in the 
modeling the possibility that people actually had consistent concepts, but they were just noisily measured, which could make it look as though people had different conceptual representations when in fact they did not. Our main results showing multiple concepts in the population therefore reflect statistical evidence of multiple concepts above and beyond response inconsistencies. Our primary analysis uses a non-parametric Bayesian clustering model in order to infer how many types of each concept (clusters) were likely to be present in our sample-for example, based on similarity judgements, how many different concepts of "finch" did people exhibit? This clustering method does not presuppose a fixed number of clusters, but infers a distribution of how many clusters are likely present based on the data. The distribution on the number of concepts combines two competing pressures: on the one hand, we should be biased to prefer a small number of clusters since this is a simpler theory. In the absence of data, the number of clusters should not be "multiplied without necessity", in the words of Ockham's Razor. Simultaneously, we should prefer a clustering which does a good job of explaining the data. Here, that means that the inferred clustering should predict responses, meaning that two individuals in the same cluster should give similar responses (see Figure 1). We use a non-parametric scheme (42-44) which translates both of these pressures into probability theory, and then balances-optimally, in a precise sense-between the two. In particular, this clustering approach (see Methods) infers a probabilistic assignment of participants to latent "concept" clusters such that there are as few clusters as possible, while still being able to adequately explain their response patterns. This inference critically depends on the reliability of subject responses and only using this model are we able to infer the number of clusters that likely generated the data.

However, we are also interested in the number of clusters present in the population beyond our experimental sample. To quantify this, we used an estimator from ecology (45). This model is more commonly used in species estimation in population ecology, where one might sample animals, observe how many of each species were collected, and estimate the total number of species present in the world (i.e. outside of the sample) from the distribution in the sample; closely related techniques can be found in $(46,47)$. Here, we use the most likely clustering of individuals to estimate the total number of concepts present in the world, outside of our sample.

Finally, we quantified people's metacognitive awareness of differences by asking participants to report what proportion of other people they expected to agree with them about their similarity judgements, and compared these reports to the observed agreement levels.

These methods allow us to test a variety of novel hypotheses about variation in human conceptual systems. First, by examining the estimated number of clusters (both in the sample and the general population), we evaluate how many measurably distinct representations can be found in the population. We note that this estimate is necessarily conservative since it is derived by similarities to a relatively small number of other nouns; larger and more detailed experiments might reveal more conceptual variation. Despite this conservativity, our results indicate that there is substantial variation present, even for these common nouns. Because this inference relies on a probabilistic model which incorporates multiple-measurement reliability, these clusters cannot be due to measurement noise. Our results also indicate common nouns and politicians have roughly the same 

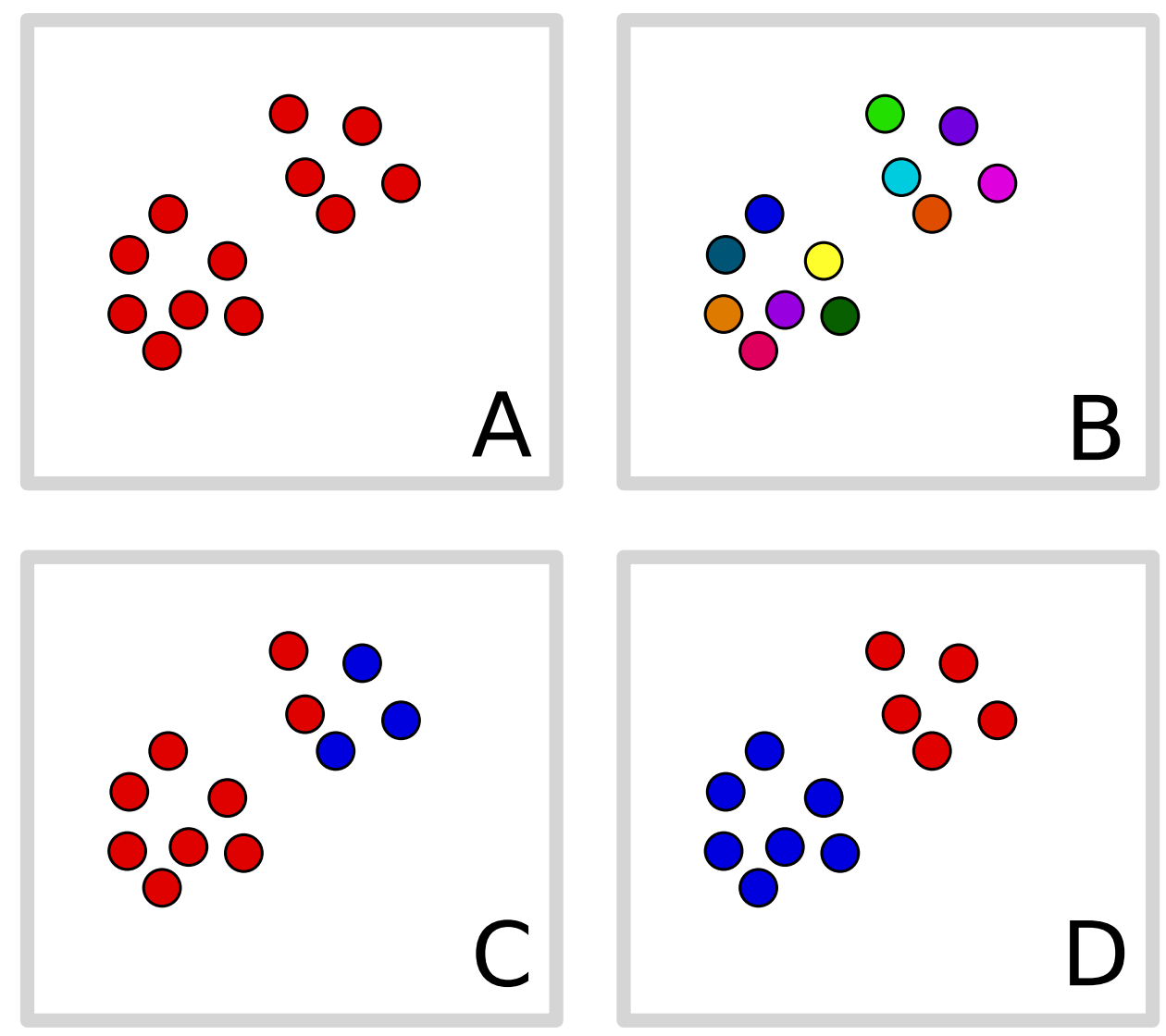

FIGURE 1: Hypothetical clustering of response vectors, here visualized in 2D. The simplest solution is to put all points into the same cluster (A), but then responses (locations) are not well-explained by clusters. If each point is in a separate cluster (B) then each point is perfectly predicted by the cluster, but the solution is complex. A compromise like (D) finds a small number of clusters that adequately explain the data. The correct clustering (D) will be preferred over alternatives even with the same number of total clusters which fit the data less well $(\mathrm{C})$.

number of different concepts in the population: both reflect substantial diversity. Finally, the meta-cognitive results show that people are generally unaware of these differences: most people expect that most others will answer the same way that they do. This lack of awareness indicates highlights that such latent variation in what words are thought to mean may underlie disagreement on broader social and political issues.

\section{Results}

\section{Experiment 1}

We recruited 1,799 participants on Amazon Mechanical Turk. Half were asked to make similarity judgements about animals and the other half to make judgements about U.S. 
politicians. Each participant was randomly assigned to a single target from one domain (e.g. "finch"), presented with 36 unique pairs of other objects in the domain, and asked which was more similar to the target. Thus, participants responded to queries such as "Which is more similar to a finch, a whale or a penguin?" Each trial was repeated for a total of 72 trials in order to measure response reliability and detect trial-by-trial idiosyncractic feautres of stimuli. To quantify metacognitive awareness of diversity, participants were also simultaneously asked on every trial to guess how many people out of 100 would agree with their response.

We coded each participant's responses to a single word as a binary vector, corresponding to the forced-choice similarity rating between every other pair of items. In modeling, we assumed that individual vectors were sampled from a collection latent clusters that specified an average response vector. We used a nonparametric Bayesian technique, a Chinese Restaurant Process (42-44), to model a posterior distribution on the number of clusters for each word independently, marginalizing out the average response vectors for each cluster and assuming a reliability given by the overall average reliability. We note that this clustering model works in the space of response vectors, not in the lowerdimensional psychological space itself; thus, our approach does not explicitly model correlations that may exist between items, but also does not require us to make assumptions about the dimensionality or metric properties of the latent space. This technique permits us to find a distribution over the number of clusters present in the population, taking into account both the reliability of individual responses and uncertainty about the latent response vector characterizing each cluster (e.g. what each participant's "finch" cluster corresponds to in terms of similarities). The model builds in a prior preference for fewer clusters - a version of Ockham's Razor-but we also present results with no such prior. The maximum a posteriori clusterings found in sampling were additionally put through a species-count estimator which estimates the true number of clusters present in the global population, beyond our finite sample size (45). This estimator uses sampled individuals which are observed to fall into a distribution of species and estimates the total number of species (here, clusters) in the population at large.

The overall subject reliability is around 90\% (see Figure 6 in Materials and Methods), indicating subjects are both not responding with random guesses, nor are they responding with ad hoc responses that vary from trial to trial. Subject responses likely reflect stable aspects of how they conceptualize these concepts throughout the context of the experiment.

Figure 2 shows a visualization of participants' similarity judgements using distributed stochastic neighbor embedding (t-SNE) (48). This technique places individual participants' response vectors in a $2 \mathrm{D}$ plane such that nearby participants give similar response vectors. The closer two points are together, the more closely their concepts align; however, these scales are relative and cannot easily be compared across plots. Points in this plot have been colored according to the maximum a posteriori assignment of participants to clusters according to the clustering model, which was run independently from t-SNE. This figure illustrates that two independent methods provide convergent characterizations of how people are distributed in the space since each color (generated according to the clustering model) tends to be in a single spatial position (generated by t-SNE). Note that 
the color assignments do not perfectly match spatial arrangements, likely due to t-SNE dimensionality reduction and different trade-offs being applied to edge-case participants by our algorithm and t-SNE.

To understand the number of concepts in the population, we first look at the posterior distribution over the number of clusters inferred. Figure 3 shows the estimated number of conceptual kinds (y-axis) for each semantic domain (subplot), as a function of the number of participants included (x-axis). This figure shows that as our sample size increases from 10 to 100 individuals per concept, the number of estimated concepts reaches 7 to 12 for politicians and 4 to 10 for animals. The maximum a posteriori clustering (in purple) and the ecological estimator (in blue) are in the range of 10-20 latent concepts in the population, and are higher for politicians than for animals. We find similar ranges even if we use a prior which is uniform on the clusterings (orange).

We note that the number of inferred concepts is not necessarily monotonically increasing in the number subjects, since additional subjects may shape the geometry of the space (e.g. providing evidence that two separate clusters are actually one wider cluster). In addition, most of the latent diversity can be found in small numbers of subjects-even distinct clusters can be found when examining 50 individuals. The point at which each subplot levels off is due to a combination of the reliability of individual responses, the number of items, and the true number of concepts in the population. However, limited reliability and a finite number of items mean that our analysis is likely to under-estimate the number of clusters.

Figure 4 shows the probability that the population contains only one concept for each word, according to the clustering model (Recall that due to the limitations of the similarity measure, this is an over-estimate). Political words are far less likely to have a single meaning than animal words, matching the patterns in the number of clusters in Figure 3. Generally, this provides strong statistical support to the idea that there are multiple meanings in the population for these terms, despite the fact that these multiple concepts all have the same word. However, if the distribution of participants to meanings tends to be heavily skewed (e.g. most participants have the same meaning), then this diversity might be relatively inconsequential. Figure 4 shows the probability that two randomly chosen individuals will have the same concept in this analysis, which is a relatively robust statistic since it depends largely on the frequency of the most common concepts for each word rather than the tails of the distribution. This agreement probability averages to around $25-50 \%$ for animals and $10-30 \%$ for politicians. This indicates that most individuals one encounters will tend to have a measurably different conceptual representation. Again, this is likely to over-estimate true rate of agreement since we only tested a small number of questions.

Most importantly, our results show that people are generally not aware of these differences. Figure 4 shows the agreement rate on responses (x-axis) compared to people's predicted estimates of agreement ( $\mathrm{y}$-axis). If people understood the population's variation in responses, the trials shown in this plot would all fall along the $y=x$ line. Instead, this figure shows that for most of the range of actual agreement (e.g. $\sim 0 \%-80 \%$ ) people tend to consistently believe that about $2 / 3$ of participants will agree with them, no matter what true proportion actually do. This is true even for the lowest agreement responses: 
most participants believe their response is in the majority even when essentially $0 \%$ of other participants agree with them. This is unlikely to be due to a failure to engage this aspect of the task because participants do reliably increase their estimates on the highest agreement items (e.g. $\sim 80 \%-100 \%$ ), which results in a reliable rank-order correlation overall (Spearman's $\rho=0.45, p<0.001$ ). The increase, though, is not well-calibrated to the population variation. Moreover, these patterns likely reflect meta-cognitive limitations (49-51) rather than differences in effort or motivation because these trials were interspersed with the main task, which had very high within-subject reliability.

\section{Experiment 2}

Experiment 2 consisted of two parts: feature elicitation and feature rating. In feature elicitation, we recruited 16 registered users on Prolific. Half of the participants were asked to list 10 single-word adjective features for each of the 10 animals in Experiment 1. The other half were asked to list 10 single-word adjective features for each of the 10 U.S. politicians in Experiment 1. We kept all features that were mentioned more than once after removing non-adjectives, inappropriate words, and typos, as well as combining synonyms.

Then, 1,000 registered users on Prolific were asked to rate either 105 animal features or 105 politician features from the feature elicitation experiments. Each participant was randomly assigned to rate features of two animals (e.g. "dolphin" and "whale") or two U.S. politicians (e.g. "George W. Bush" and "Hillary Clinton"). Participants were asked questions such as "Is a finch smart?" and responded by clicking either the "Yes" or "No" button on the screen. Each question was asked twice to measure response reliability. Thus, each participant saw 420 question trials.

Participant reliability was high with an average reliability of $86 \%$ during feature rating, indicating participants were not responding with random guesses. Similar to Experiment 1 , subject responses likely reflect stable aspects of subjects' conceptual representations.

Clustering participants based on their feature ratings serves as a conceptual replication of Experiment 1. The number of concepts found is 6 to 11 for politicians 5 to 8 for animals, compared to 7 to 12 for politicians and 4 to 10 for animals in Experiment 1 (see Figure 10 in SM). Likewise, the ecological estimator results in 8 to 30 latent concepts in the population, compared to 10 to 20 in Experiment 1. Such similar findings, despite a very different paradigm, provides convergent support for conceptual diversity.

Figure 5 shows mean agreement for a sample of features and concepts. Many features (in yellow) show near universal agreement among participants. A similar number of features show large disagreement among participants (in orange). Most participants agree that seals are not feathered but are slippery while disagreeing as to whether they are graceful. Likewise, most participants agree that Trump is not humble and is rich, but there is high disagreement as to whether he is interesting. These sorts of disagreements reflect the different conceptual representations possessed by our participants. 


\section{Discussion}

We report statistical evidence of more than one variant of concepts in the population. In fact, we find that most people the average language user meets will not share their same concept. These results are unexpected in part because the measures we used are coarse. If one could gather an arbitrary amount of data, one might expect to find small differences between people: one interlocutor might have specific memories that make their representation idiosyncratic, perhaps different from anyone else. However, our experimental approach was based on judging similarities and features-not an exhaustive inventory of each person's memories or associations - and we were nonetheless able to statistically justify measurably distinct representations, even for common nouns. If differences can be detected with these methods, it indicates that there is substantial variation in the population for lexical meanings. This variation exists despite the fact that the people use the same word for each concept, and people are relatively unaware that others will tend to give differing similarity judgements.

However, our results do not support the notion that every single use of a concept is distinct or entirely idiosyncratic (52): subjects did group into clusters and did provide highly reliable responses across trials. We emphasize, though, that studies with more items, or items that focus more on corner cases, might find greater diversity than reported here. Moreover, the subject pool in our experiment was relatively homogeneous, and future studies of cultural differences may point to more diversity in word usage based on diversity of experience (29). Indeed, while our method allows us to quantify conceptual diversity, it does not pinpoint what specific representational differences drive this diversity. These differences may indeed go deep with respect to theories and interrelations between the concepts studied and others (53-55).

In general, theories of word learning and conceptual development will need to work out how human language users acquire distinct representations for shared words. In turn, theories of communication and language use (e.g. $(56,57))$ will need to address both differences in word referents, and lack of awareness of those differences. People's general obliviousness to variation has important implications for productive discourse structure. Fundamental misunderstandings may originate with individuals using the same word for distinct conceptual representations. Indeed, such differences in word meanings might underlie many classic philosophical questions (58). Generally, our results may help to explain why "talking past each other" appears to be common in social and political debates: the common ground of even the most basic word meanings is only imperfectly shared.

\section{Materials and Methods}

Experiment 1 was run using a custom built web interface on Amazon Mechanical Turk on 8/20/19 through 8/22/19 (animals) and 9/11/19 through 9/12/19 (politicians). Participants were required to be from the U.S. and have a minimum $95 \%$ approval rating from previous tasks. Experiment 2 was run on 04/23/21 through 05/09/21 (animals) and 05/13/21 through 05/17/21 (politicians) using Prolific and Qualtrics. Participants were all above 18 years old, fluent English speakers, and physically present in the United States based on 
pre-screening questions. Responses were recorded on a secure server and no participants were excluded from data analysis.

\section{Clustering Methods}

Responses were clustered using a non-parametric, Bayesian clustering model, a "Chinese restaurant proces" (44), with a custom implementation in Python. If $x=\left\langle x_{1}, x_{2}, \ldots, x_{k}\right\rangle$ denotes the number of subjects at cluster $n$ subjects have each concept (for a given word), this model assigns $x$, a partition on individuals, a prior of

$$
\frac{1}{n !} \prod_{i=1}^{k}\left(x_{i}-1\right) \text { !. }
$$

Within each cluster, we use a Beta-Bernoulli likelihood where subjects assigned the same cluster are assumed to generate the same latent vector of answer probabilities. We marginalized out each cluster's probability vector. Thus, if $a_{i j}$ and $b_{i j}$ are the number of each type of response to question $j$ in cluster $i$, then the marginal likelihood of those responses is,

$$
\frac{\Gamma(2 \alpha)}{\Gamma(\alpha)^{2}} \cdot \frac{1}{\Gamma\left(x_{i}+2 \alpha\right)} \prod_{j=1}^{x_{i}} \frac{\Gamma\left(a_{i j}+\alpha\right)}{\Gamma\left(a_{i j}+1\right)} \cdot \frac{\Gamma\left(b_{i j}+\alpha\right)}{\Gamma\left(b_{i j}+1\right)} .
$$

Here, $\alpha$ characterizes the noise level assumed by the likelihood. We set the single likelihood parameter $\alpha=0.16$ such that two samples from a Bernoulli with parameter $p \sim \operatorname{Beta}(\alpha, \alpha)$ agreed with each other with probability 0.88 , which is proportion of time subjects' second and first responses agreed (analysis of the dependence of the results to the assumed $\alpha$ is in $\mathbf{S M}$ ).

Inference was run using a Gibbs sampler, using both a the prior (1) above and a uniform prior. All runs used the same likelihood (2). The Gibbs sampler followed standard inference techniques by selecting each individual and resampling a cluster assignment for them based on the posterior probability of each possible cluster assignment (including assigning to a new cluster). The sampler was initialized with a configuration where each individual started in the same cluster. These sampling methods require iterations of burnin before they converge to a stable set posterior distribution. We assessed convergence using multiple runs and ensured that chains arrived at the same solution. Figure 7 in SM shows the convergence of three chains for each concept over 300k iterations. Our final run used a 100k iterations of burn-in and an additional 200k iterations of sampling.

\section{Ecological estimator}

Finally, we use an ecological estimator from (45), extending a previous estimator (59), in order to approximate the total number of concepts in the population. This estimator uses the total number of observed clusters (concepts) and the total number of sampled individuals in order to estimate how many concepts were likely unobserved. Like, for instance, Good-Turing estimation (46), this estimation depends on the number of clusters containing a single person, but also includes additional terms. Let $f_{i}$ denote the number 
of clusters containing $i$ individuals in the maximum a posteriori Bayesian clustering. The estimator is based on $\hat{S}_{C h a 1}$, given by,

$$
\hat{S}_{\text {Chao } 1}= \begin{cases}S_{o b s}+\frac{(n-1)}{n} \frac{f_{1}^{2}}{2 f_{2}}, & \text { if } f_{2}>0 \\ S_{o b s}+\frac{(n-1)}{n} \frac{f_{1}\left(f_{1}-1\right)}{2}, & \text { if } f_{2}=0 .\end{cases}
$$

Here, $S_{o b s}$ denotes the number of observed clusters and $n$ is the number of participants sampled. The estimator we used adjusts $\hat{S}_{\text {Chao } 1}$ to yield $\hat{S}_{i C h a o 1}$,

$$
\hat{S}_{i \text { Chao } 1}=\hat{S}_{\text {Chao } 1}+\frac{(n-3)}{n} \cdot \frac{f_{3}}{4 f_{4}} \cdot \max \left(f_{1}-\frac{(n-3)}{(n-1)} \frac{f_{2} f_{3}}{2 f_{4}}, 0\right) .
$$

\section{References}

1. F. E. Zachos, Species concepts in biology, vol. 801 (Springer, 2016).

2. K. Stotz, P. E. Griffiths, R. Knight, Studies in History and Philosophy of Science Part C: Studies in History and Philosophy of Biological and Biomedical Sciences 35, 647 (2004).

3. E. N. Trifonov, Journal of Biomolecular Structure and Dynamics 29, 259 (2011).

4. E. M. Grossnickle, Educational Psychology Review 28, 23 (2016).

5. K. Lehrer, Theory of knowledge (Routledge, 2018).

6. R. J. Sternberg, Intelligence. (Cambridge University Press, 2005).

7. E. P. Wigner, Philosophical Reflections and Syntheses (Springer, 1995), pp. 225-244.

8. E. de Waal, S. ten Hagen, Physics Perspectives 22, 55 (2020).

9. R. Benjamin, Race after technology: Abolitionist tools for the new Jim code (Cambridge University Press, 2019).

10. P. Stewart, US Rep 378, 184 (1964).

11. O. A. Leshem, E. Halperin, Proceedings of the National Academy of Sciences (2020).

12. A. Winkler, Gunfight: The battle over the right to bear arms in America (WW Norton \& Company, 2011).

13. N. Goldfarb, Annual Review of Linguistics 7, 473 (2021).

14. W. Labov, New ways of analyzing variation in English (1973).

15. M. E. McCloskey, S. Glucksberg, Memory \& Cognition 6, 462 (1978).

16. W. S. Torgerson, Psychometrika 17, 401 (1952).

17. R. N. Shepard, Psychometrika 27, 125 (1962). 
18. R. N. Shepard, Science 210, 390 (1980).

19. L. R. Tucker, S. Messick, Psychometrika 28, 333 (1963).

20. V. E. McGee, Multivariate Behavioral Research 3, 233 (1968).

21. J. D. Carroll, J.-J. Chang, Psychometrika 35, 283 (1970).

22. L. E. Bush, Journal of personality and social psychology 25, 50 (1973).

23. Y. Takane, F. W. Young, J. De Leeuw, Psychometrika 42, 7 (1977).

24. L. Bocci, M. Vichi, Psychometrika 76, 691 (2011).

25. K. Okada, M. D. Lee, Journal of Mathematical Psychology 70, 35 (2016).

26. A. Gelman, et al., Bayesian data analysis (CRC press, 2013).

27. J. K. Kruschke, Wiley Interdisciplinary Reviews: Cognitive Science 1, 658 (2010).

28. C. Starmans, O. Friedman, Cognitive Science 44, e12850 (2020).

29. H. H. Clark, Context in language learning and language understanding 6387 (1998).

30. A. Shtulman, I. Share, R. Silber-Marker, A. R. Landrum, Cognitive Development 55, 100895 (2020).

31. E. Sumner, E. DeAngelis, M. Hyatt, N. Goodman, C. Kidd, PloS one 14, e0217207 (2019).

32. S. Laurence, E. Margolis, Concepts: core readings 3, 81 (1999).

33. R. N. Shepard, Psychometrika 27, 219 (1962).

34. T. K. Landauer, S. T. Dumais, Psychological review 104, 211 (1997).

35. T. Mikolov, K. Chen, G. Corrado, J. Dean, arXiv preprint arXiv:1301.3781 (2013).

36. J. Graham, J. Haidt, B. A. Nosek, Journal of personality and social psychology 96, 1029 (2009).

37. J. A. Frimer, Journal of Research in Personality 84, 103906 (2020).

38. A. Tversky, I. Gati, Cognition and categorization. Hillsdale, Erlbaum (1978).

39. D. L. Medin, R. L. Goldstone, D. Gentner, Psychological review 100, 254 (1993).

40. D. Gentner, A. B. Markman, American psychologist 52, 45 (1997).

41. A. B. Markman, D. Gentner, Cognitive psychology 25, 431 (1993).

42. S. J. Gershman, D. M. Blei, Journal of Mathematical Psychology 56, 1 (2012).

43. J. R. Anderson, Psychological review 98, 409 (1991). 
44. J. Pitman, Probability theory and related fields 102, 145 (1995).

45. A. Chao, C.-H. Chiu, eLS pp. 1-11 (2016).

46. I. J. Good, Biometrika 40, 237 (1953).

47. W. A. Gale, G. Sampson, Journal of quantitative linguistics 2, 217 (1995).

48. L. v. d. Maaten, G. Hinton, Journal of machine learning research 9, 2579 (2008).

49. A. Gopnik, A. N. Meltzoff, P. Bryant, Words, thoughts, and theories, vol. 1 (Mit Press Cambridge, MA, 1997).

50. H. Wimmer, J. Perner, Cognition 13, 103 (1983).

51. A. I. Goldman, et al., Simulating minds: The philosophy, psychology, and neuroscience of mindreading (Oxford University Press, 2006).

52. D. Casasanto, G. Lupyan, The conceptual mind: New directions in the study of concepts pp. 543-566 (2015).

53. G. L. Murphy, D. L. Medin, Psychological review 92, 289 (1985).

54. D. L. Medin, L. J. Rips, Concepts and Categories: Memory, Meaning, and Metaphysics. (Cambridge University Press, 2005).

55. S. A. Gelman, C. H. Legare, Annual review of anthropology 40, 379 (2011).

56. D. Wilson, D. Sperber, Relevance theory (2002).

57. H. P. Grice, Studies in the way of words (Harvard University Press, 1989).

58. S. T. Piantadosi, Mathematics, Substance and Surmise (Springer, 2015), pp. 305-320.

59. R. K. Colwell, J. A. Coddington, Philosophical Transactions of the Royal Society of London. Series B: Biological Sciences 345, 101 (1994).

\section{Author contributions}

Conceptualization: Author1, Author4, Author3

Methodology: Author1, Author4, Author3

Investigation: Author1, Author2

Software: Author1, Author3

Formal analysis: Author1

Visualization: Author1, Author3, Author2

Writing - original draft preparation: Author1

Writing - review and editing: Author1, Author4, Author3, Author2

Supervision: Author4, Author3

Funding acquisition: Author4, Author3 


\section{Competing interests:}

Authors declare that they have no competing interests. 

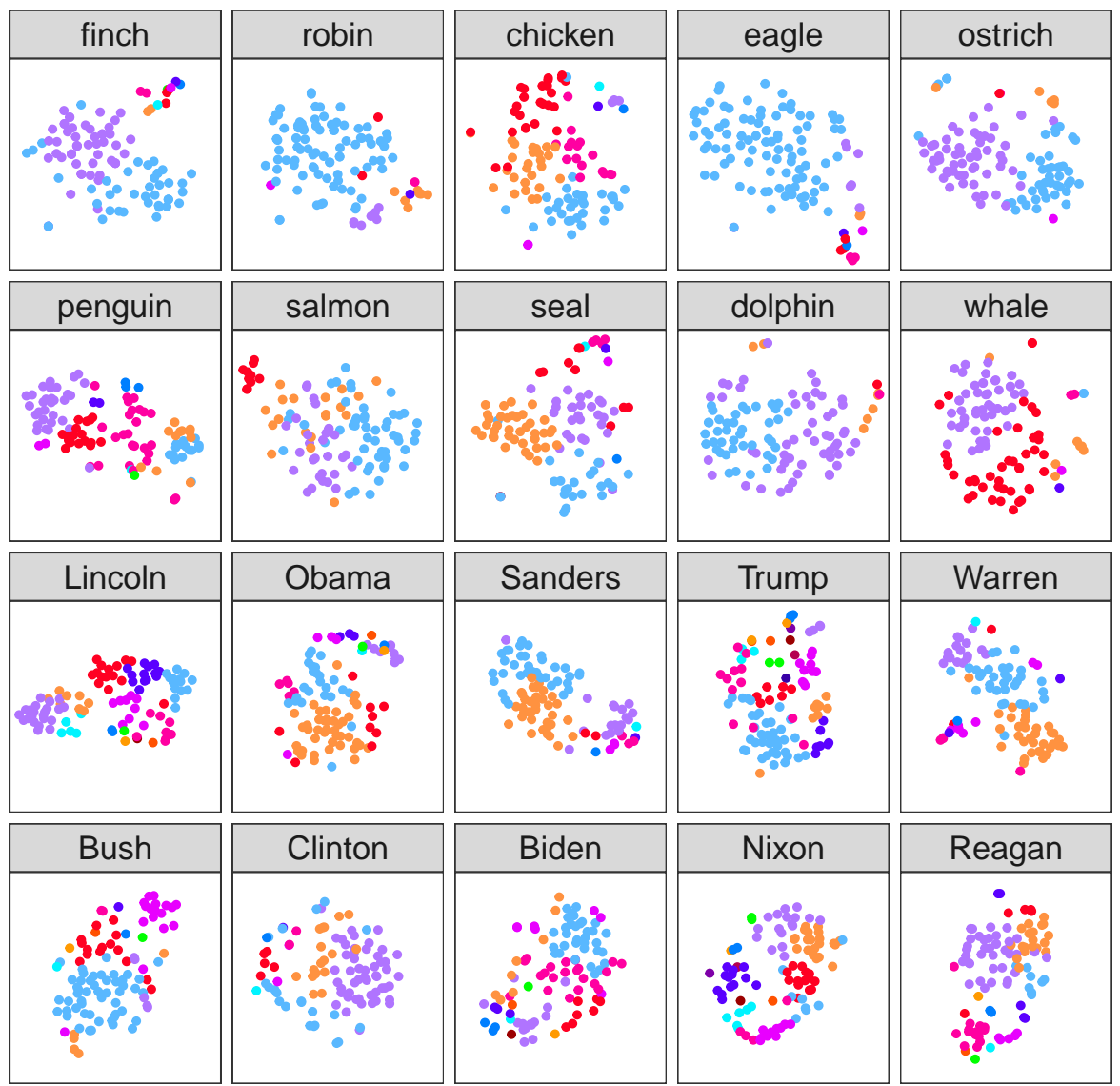

FIGURE 2: Distances between participants' conceptual representations of each target concept using distributed stochastic neighbor embedding. In this visualization, the distances between two points approximate the distance between their full rating vectors. Each plot is on the same scale. Additionally, each data-point is colored with the cluster they were assigned to in our clustering analysis, showing that the t-SNE clustering finds similar groupings. 


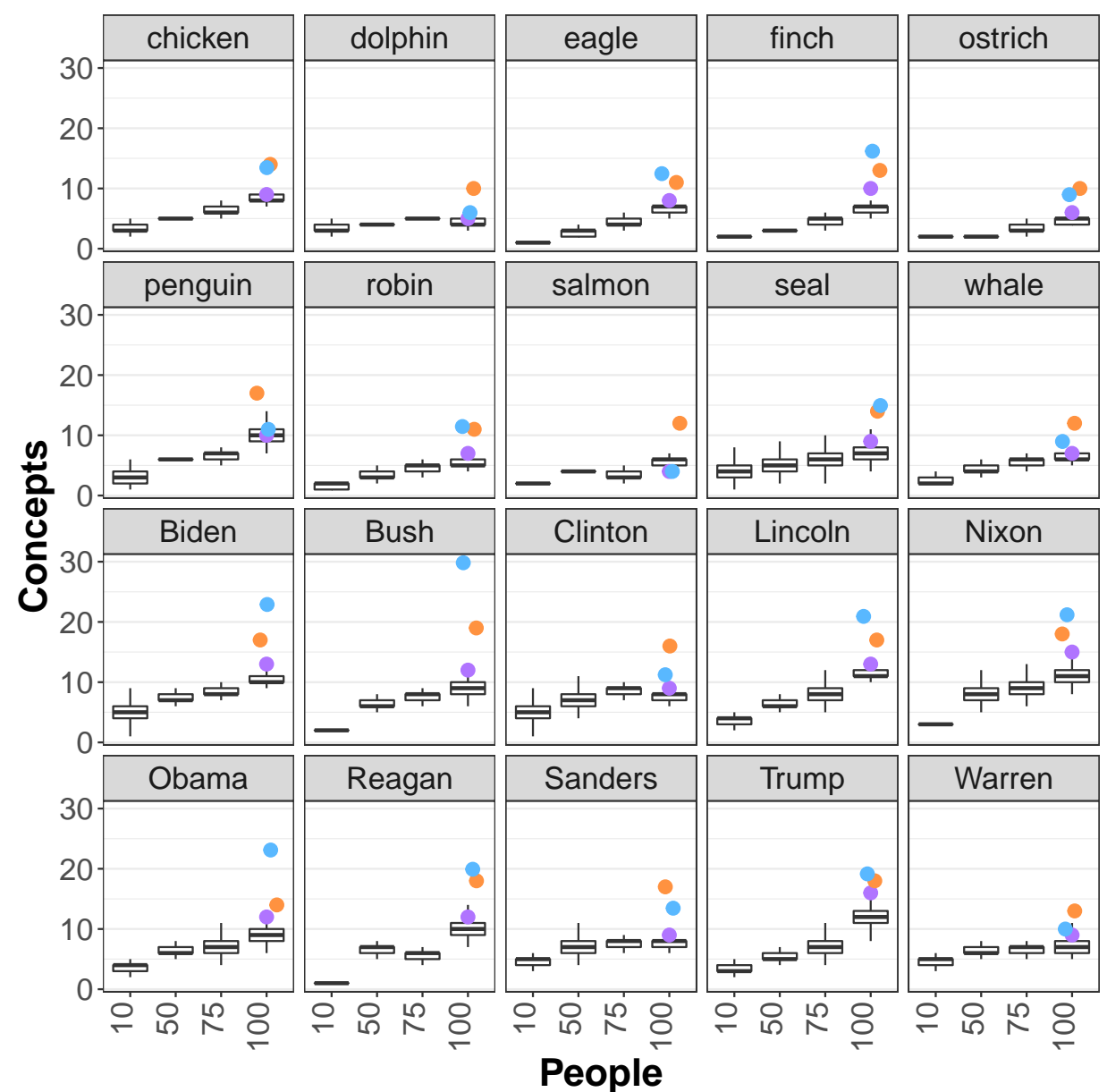

FIGURE 3: Estimated true number of concepts (y-axis) depending on the number of people sampled (x-axis). Boxes show the median 50\% quantiles of the number of unique concepts. Purple data points are the number of tables for the maximum a posteriori clustering. Orange data points are the number of tables for the MAP clustering with a uniform prior. Blue data points are a lower bound on the number of concepts estimated by the ecological estimator using the MAP clustering. 

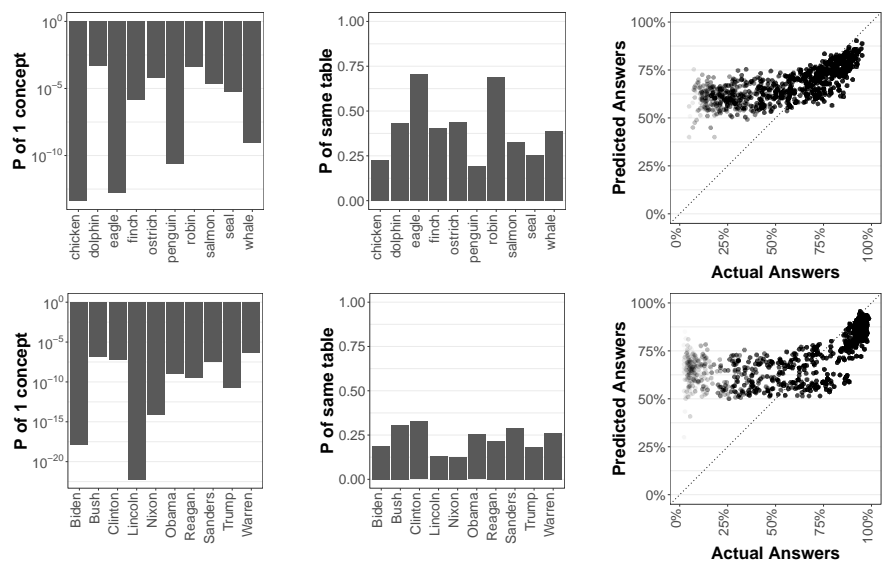

FIGURE 4: Left: Probabilities that only a single conceptual representation for each word exists, with log-axis, showing essentially zero probability for any word. Middle: Probability that a random individual will share one's own cluster for any word, showing generally low rates of agreement. Right: Predicted answers (y-axis) vs. actual answers (x-axis), showing people tend to over-estimate others' rate of agreement compared to the truth (line $y=x$ ). 


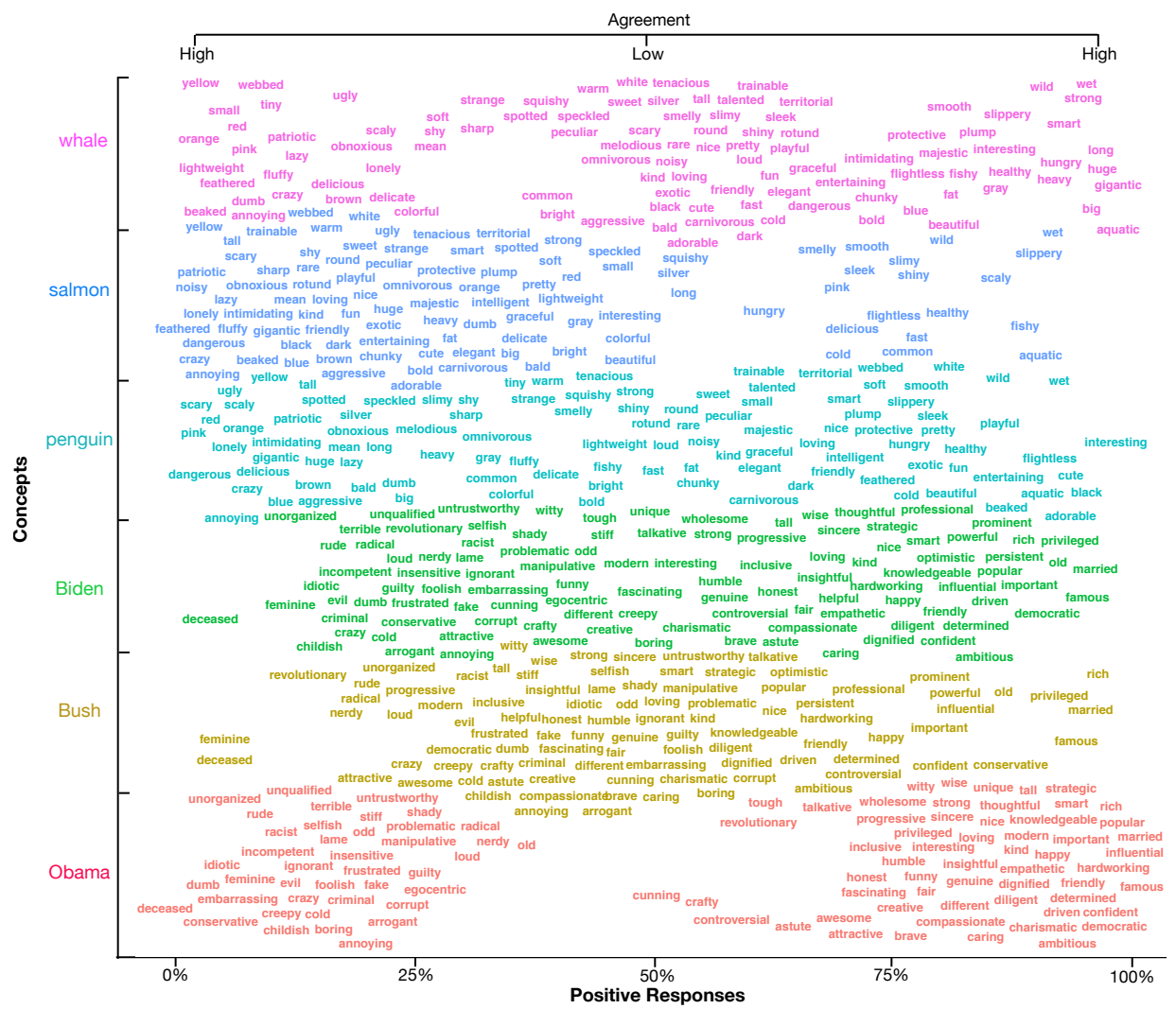

FIGURE 5: A sampling of feature responses for 3 animals and 3 politicians (y-axis). The $\mathrm{x}$-axis plots the mean percentage of "yes" responses for a given feature. Features on the left are generally agreed to not apply and features on the right are usually agreed to be applicable. Features in the center however, show high disagreement among participants and are the primary features responsible for differing conceptual representations among participants. 


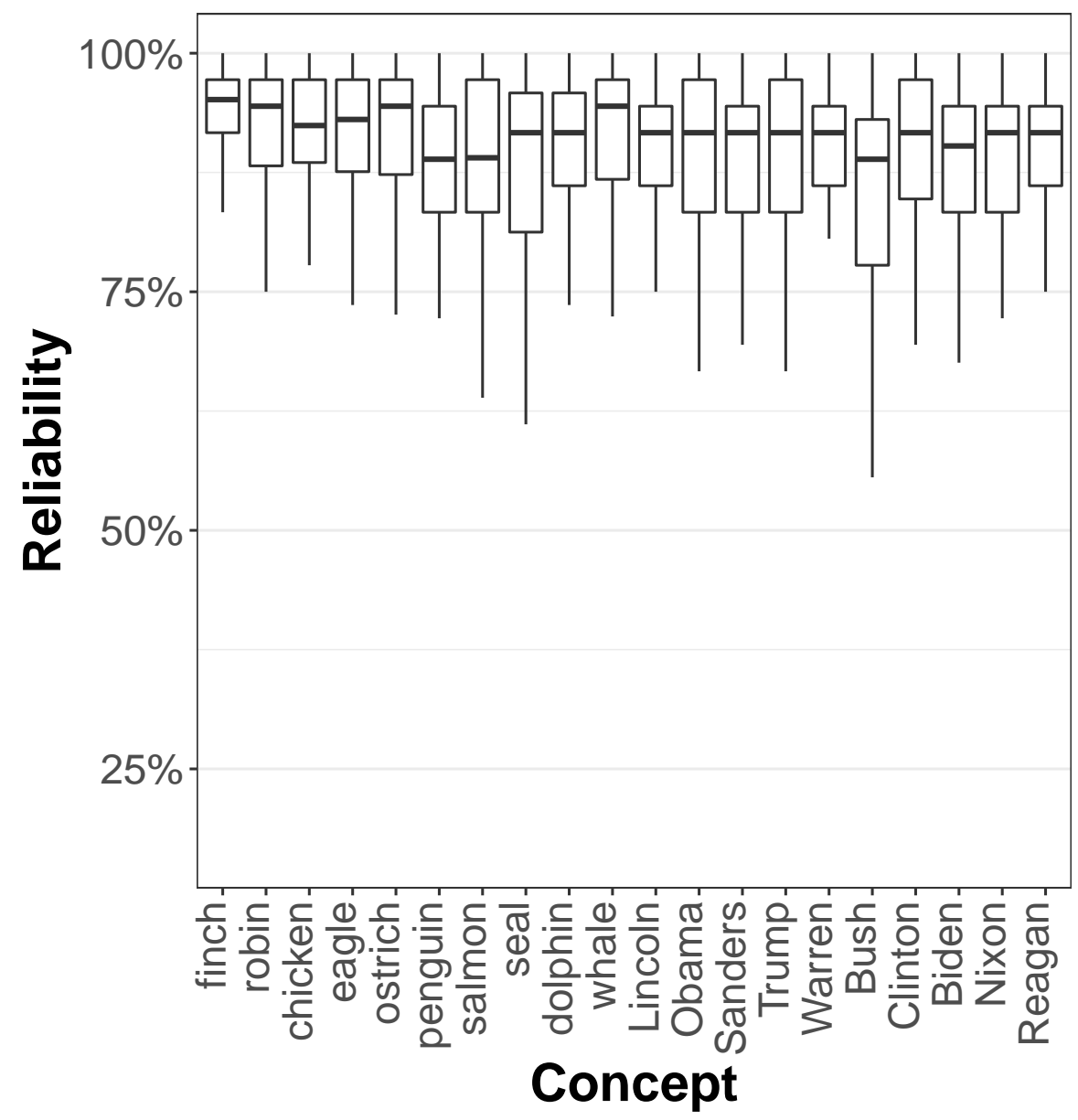

FIGURE 6: Participant reliability scores for each concept in Experiment 1. Boxes show the median 50\% reliability quantiles. Median reliabilities for concepts range from $88.8 \%$ to $94.4 \%$

\section{Supplemental Materials}

\section{Robustness to $\alpha$}

In order to assess the robustness of our general results to the value of $\alpha$, we also ran our model using alpha values of half $(\alpha=.08$, reliability $=93 \%)$ and double $(\alpha=.32$, reliability $=80 \%$ ) the value used in the main results. As Figure 8 and Figure 9 show, results are not substantially different, providing evidence that our main results are not sensitive to participant reliability. Specifically, our $87 \%$ observed reliability yielded $4-10$ concepts for animals and $7-12$ for political figures. Increasing reliability to $93 \%$ resulted in $5-9$ for animals and $7-11$ for politicians. Decreasing reliability to $80 \%$ resulted in $3-12$ for animals and $7-12$ for politicians. 


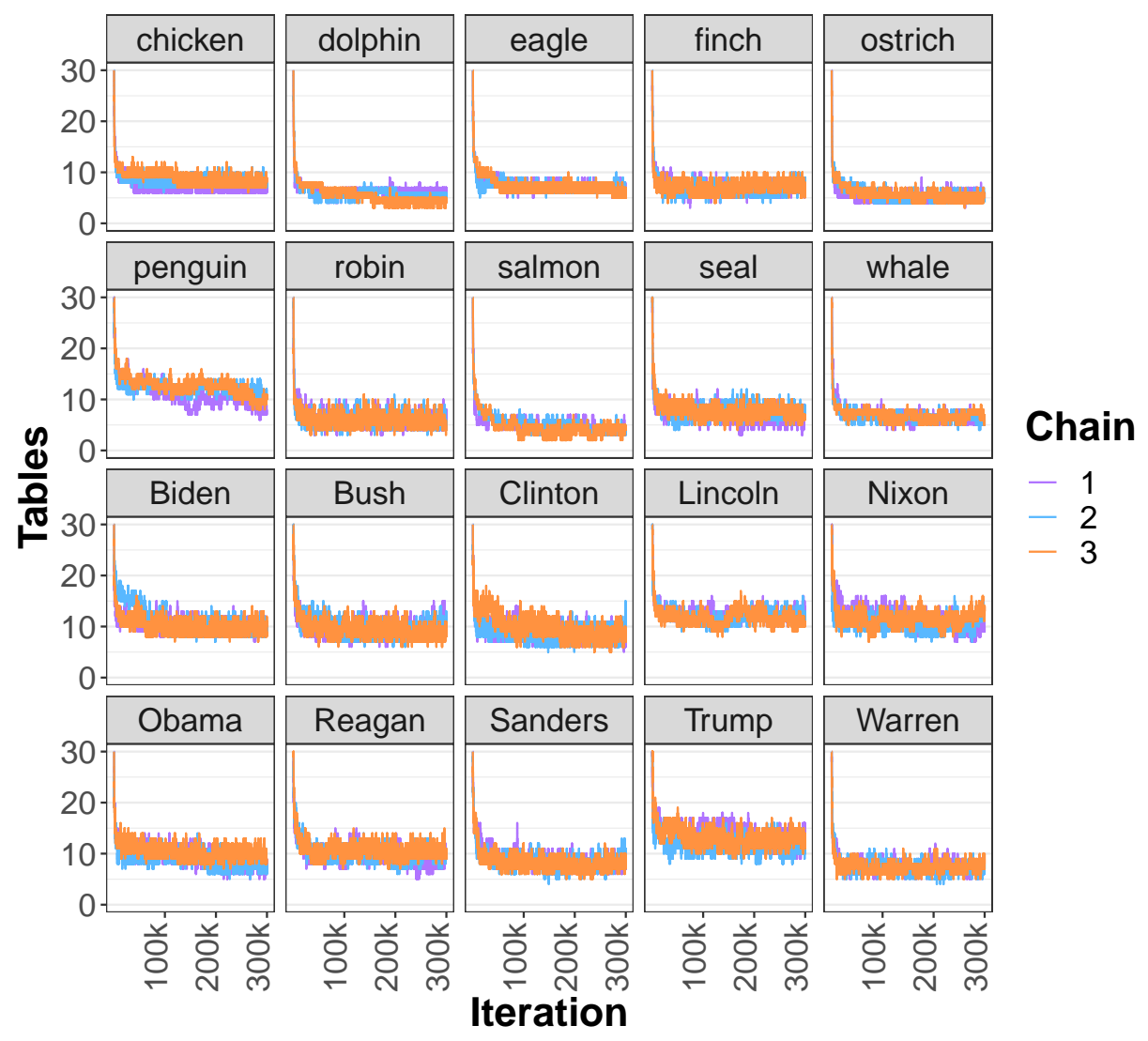

FIGURE 7: Convergence of the clutsering model was assessed with multiple runs. Chains converge between 10,000 and 50,000 iterations. 


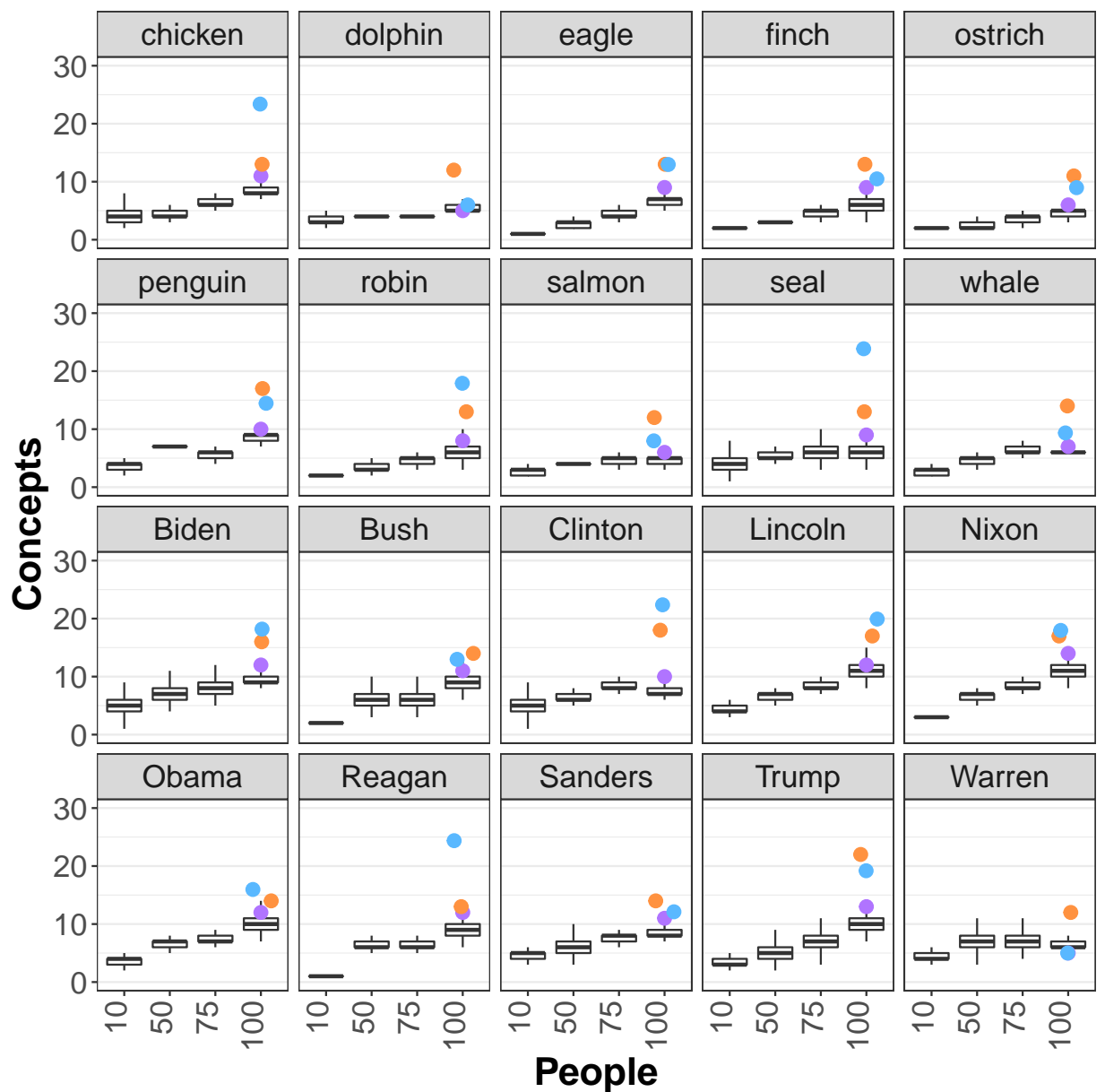

FIGURE 8: Estimated true number of concepts (y-axis) depending on the number of people sampled (x-axis) for $\alpha=.08$, reliability $=93 \%$ 


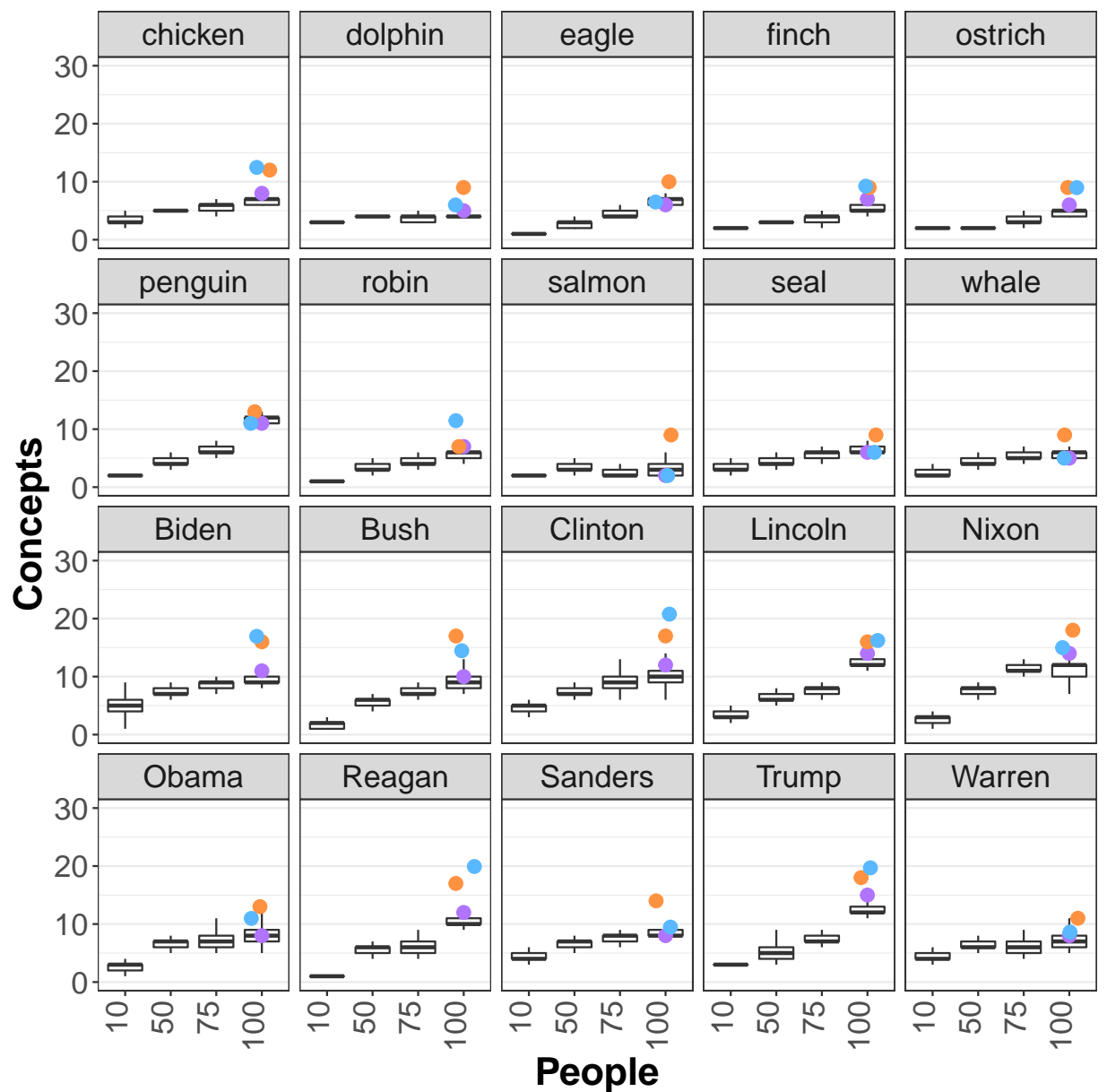

FIGURE 9: Estimated true number of concepts (y-axis) depending on the number of people sampled (x-axis) for $\alpha=.32$, reliability $=80 \%$ 


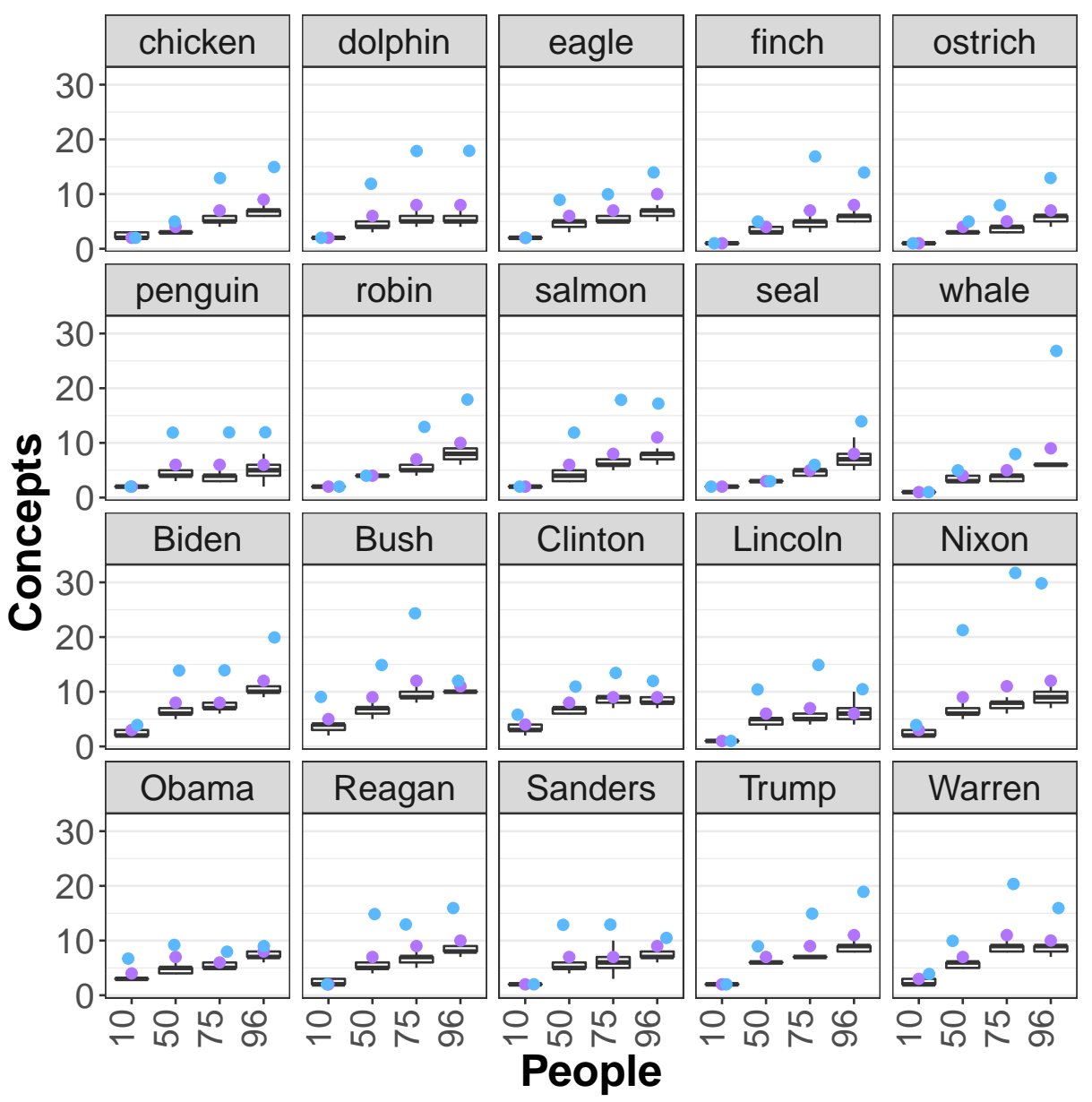

FIGURE 10: Experiment 2 - Estimated true number of concepts (y-axis) depending on the number of people sampled (x-axis). Purple data points are the number of tables for the maximum a posteriori clustering. Blue data points are a lower bound on the number of concepts estimated by the ecological estimator using the MAP clustering 